\title{
Analyzing the Empirical Course of Forgetting
}

\author{
John T. Wixted \\ University of California, San Diego
}

\begin{abstract}
An analysis of the temporal properties of forgetting can be performed whenever memory is assessed at more than one retention interval. With regard to the singular property of forgetting rate, however, any theoretical interpretation is complicated by the possibility of a nonlinear scale relating a given performance measure (e.g., number of items recalled) to an underlying process. Because that scaling relationship is generally unknown, many conclusions about the rate of forgetting based on a performance measure cannot be assumed to reflect the memory process of interest. In an effort to deal with that problem, several methods of analysis have been proposed that generally yield opposing conclusions about whether a higher degree of learning results in a slower rate of forgetting. The present article describes four properties of empirical forgetting functions that may, at different times, be taken to represent the rate of forgetting and illustrates the potentially confusing practice of assigning the same verbal label to each. The difference between performance-based and psychological views of forgetting and the role of that distinction in determining one's approach to the analysis of empirical forgetting functions are also considered.
\end{abstract}

A subject of long-standing interest in the field of learning and memory is the temporal course of forgetting. Recent studies concerned with this issue have examined the form of forgetting functions spanning as few as $15 \mathrm{~s}$ to as many as $\mathbf{5 0}$ years (Bahrick, 1984; Modigliani, 1976; Rundquist, 1983, 1986; Slamecka \& Katsaiti, 1988; Slamecka \& McElree, 1983; Squire, 1989; Underwood, 1964; Wickelgren, 1972, 1974). Although they are relatively easy to produce, the proper interpretation of experimental forgetting functions has proven to be a surprisingly complex undertaking. Several years ago, Slamecka (1985) and Loftus (1985a, 1985b) exchanged views on how to decide whether the rate of forgetting in one condition differs from that in another. Unfortunately, more often than not, the methods they proposed lead to opposing conclusions. After reviewing the same literature, for example, Slamecka (1985) concluded that variations in the degree of learning generally have no effect on forgetting rate, whereas Loftus (1985a) reached the incompatible conclusion that a higher degree of learning virtually always results in a slower rate of forgetting.

Bogartz (1990) found fault with both methods of analysis and proposed yet another approach based on Anderson's (1982) functional measurement theory. For entirely different reasons, Bogartz arrived at the same conclusion as Slamecka regarding the effects of learning on forgetting. Without some agreement on the proper approach to describing and interpreting the phenomenon of forgetting, a thorough analysis of the factors that alter its temporal course may be difficult to come by. In what follows, I attempt to work toward a resolution of the ambiguities surrounding the analysis of forget-

Preparation of this article was supported by National Science Foundation Grant BNS-8907936. I thank Geoffrey Loftus, Donald Bamber, Richard Bogartz, Allen Osman, and especially Ebbe Ebbesen for their thoughtful input.

Correspondence concerning this article should be addressed to John T. Wixted, Department of Psychology, C-009, University of California, San Diego, La Jolla, California 92093. ting, beginning with a brief overview of the contrasting methods of analysis.

\section{Vertical Versus Horizontal Parallelism}

Consider a simple experiment in which one group of subjects (high) is provided with a greater number of learning trials than another (low). Figure 1 (top panel) shows a hypothetical forgetting function for each group; the low-learning group clearly exhibits a reduced level of recall at all delay intervals. Of particular interest here is whether or not forgetting is also faster in the low condition. To answer this question, Slamecka and his colleagues (Slamecka, 1985; Slamecka \& Katsaiti, 1988; Slamecka \& McElree, 1983) prefer the traditional method of performing an analysis of variance to evaluate the possibility of an interaction between the degree of learning and the retention interval. If the interaction is significant, then the two conditions are said to exhibit different rates of forgetting. If not, then they are said to exhibit the same rates of forgetting. This method of analysis essentially tests whether or not the vertical distance between the two forgetting curves remains constant as a function of time (i.e., whether or not the two curves are vertically parallel). Because the vertical distance between the two functions shown in the top panel of Figure 1 remains constant as the retention interval increases, the rate of forgetting in the high condition is judged to be the same as that in the low condition.

Loftus (1978, 1985a, 1985b) argued that this approach entails an inherent scaling problem that can give rise to the conclusion of equal forgetting rates when, in fact, different rates prevail (and vice versa). Specifically, in the absence of any information regarding the way in which the underlying memory process maps onto the dependent measure in use, many statistical interactions (or lack thereof) are not definitive. Consider, for example, the venerable theory of memory which states that performance is a function of the strength of the underlying memory trace. For purposes of illustration, let us assume more specifically that the probability of recall is 

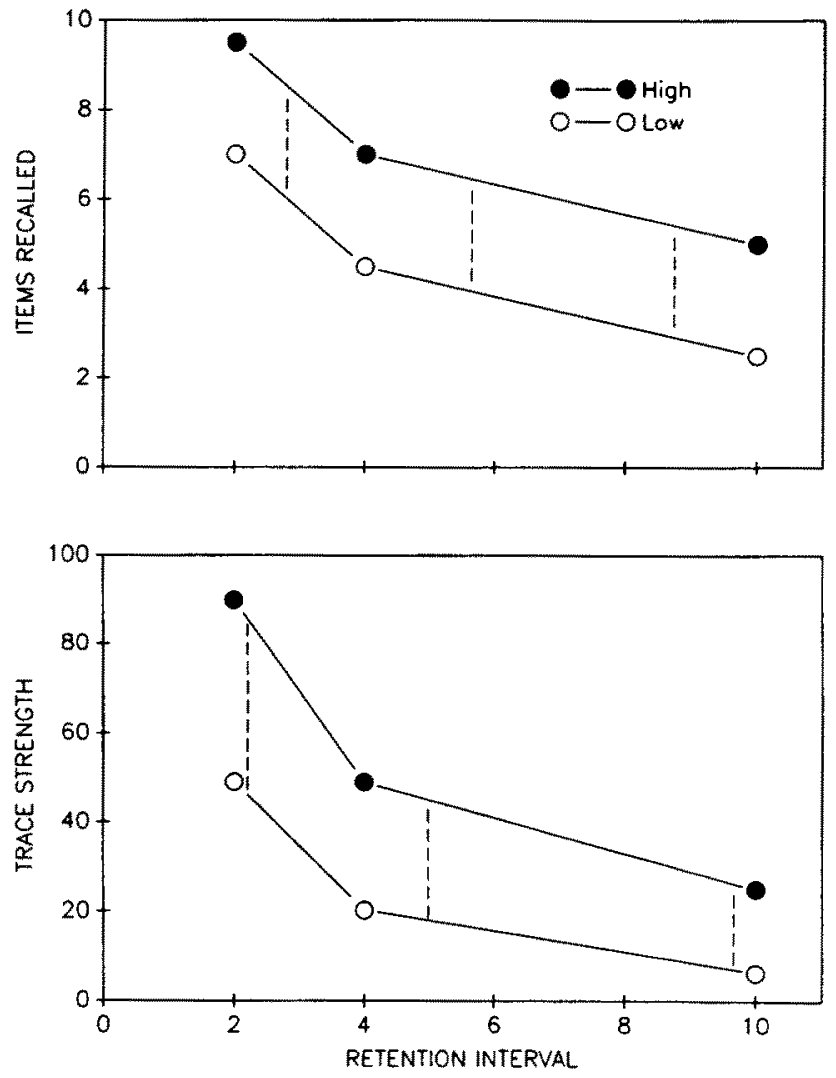

Figure 1. The top panel shows hypothetical forgetting functions produced by subjects exposed to high and low degrees of learning. The dashed lines illustrate the constant vertical separation between the two functions. The bottom panel shows forgetting functions yielded by squaring the data in the upper panel to provide a hypothetical measure of trace strength. The dashed lines illustrate the continuously decreasing vertical separation between the two functions.

equal to the square root of the item's trace strength measured, say, in hypothetical units of neural activity. In that case, a truer measure of memory would be provided by first squaring the obtained free-recall data. Applying that transformation to the data depicted in the top panel of Figure 1 yields the functions shown in the lower panel. The transformed functions are obviously nonparallel in the vertical direction, suggesting different rates of forgetting after all.

In practice, the exact form of the underlying mapping relation is always unknown and therein lies the problem. Regardless of whether the two forgetting functions are vertically parallel, they may or may not reflect equal rates of forgetting depending on the specific form of the unknown scaling function (Krantz \& Tversky, 1971; Loftus, 1978). To solve this problem, Loftus (1985a, 1985b) proposed a new way to decide whether forgetting in one condition is faster or slower than forgetting in another.

Consider a hypothetical exponential forgetting function, $a e^{-b T}$, where $T$ is the size of the retention interval, $a$ is the $y$ intercept and $b$ is the decay constant. Figure 2 depicts two exponential forgetting curves associated with different values of $a$ (reflecting different degrees of original learning), but the same decay constant, $b$. Exponential functions with identical decay constants have equivalent half-lives (i.e., they require the same amount of time to fall halfway through their full range of performance). Nevertheless, these two functions are obviously nonparallel in the vertical direction.

Loftus observed that the two curves shown in Figure 2 are parallel in the horizontal direction. That is, the horizontal distance between them remains constant as a function of time, reflecting the fact that both curves have the same halflife. By contrast, if a higher level of original learning had also slowed the half-life of forgetting (i.e., decreased $b$ ), the difference between the two curves would be reflected by an ever increasing horizontal discrepancy. Thus, a new way to gauge whether or not two functions exhibit the same rate of forgetting is to test them for parallelism in the horizontal direction. The advantage of the horizontal method is that it is immune to the scaling problems that plague the usual test of vertical parallelism. Its special immunity derives from the fact that any transformation of the dependent measure (e.g., squaring the data) adjusts the curves in the $y$ (i.e., vertical) direction only and leaves differences in the $x$ (i.e., horizontal) direction intact.

Depending upon which method of analysis is used, the same data comparing the forgetting functions of two conditions can be construed as supporting the hypothesis of different forgetting rates and identical forgetting rates! The curves depicted in Figure 2 graphically illustrate the apparent contradiction. The traditional approach clearly suggests different rates of forgetting because the vertical difference between the two functions changes continuously. The new horizontal approach, on the other hand, suggests that they are the same.

\section{Forgetting Function Properties}

Loftus and Slamecka acknowledged that their disagreement rests fundamentally on different definitions of forgetting rate.

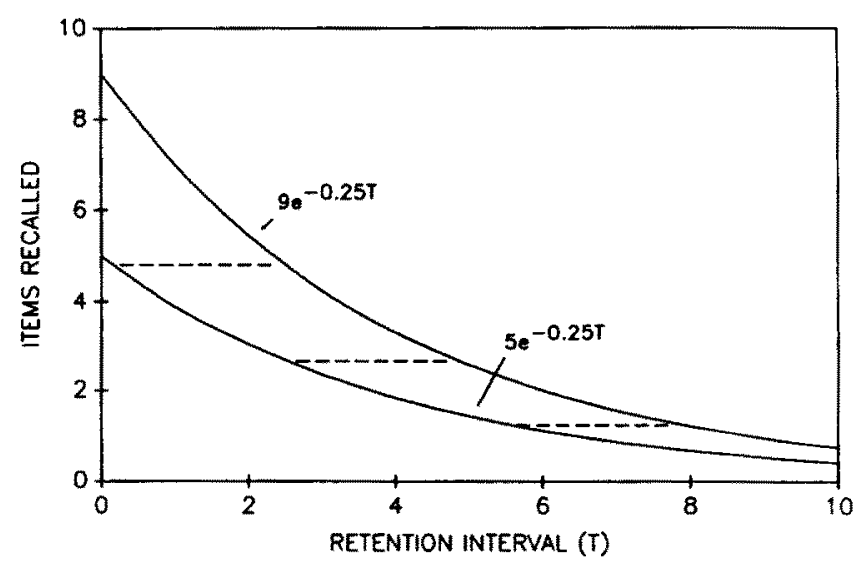

Figure 2. Idealized exponential forgetting curves representing different degrees of learning but equal decay constants. (The dashed lines illustrate the constant horizontal separation between the two curves.) 
According to one definition, the degree of learning is independent of the rate of forgetting, whereas according to the other, the degree of learning and rate of forgetting are related (Loftus, 1985b, p. 402). However, a more precise statement may be that they disagreed about the significance of scaling considerations, and for that reason chose to focus on different properties of empirical forgetting functions. It is, to some extent, misleading to assign the same verbal label to each of these properties (viz., rate of forgetting) and to then suggest that they disagree about that. At least four properties of empirical forgetting functions have served at one time or another as the basis for claims about forgetting rate. Two of these involve a measure of change in performance (absolute or relative) with respect to a fixed amount of time. The other two involve a measure of the time required to fall through a fixed (absolute or relative) range of performance.

\section{Performance Over Time}

The most widely used measure of the first kind, and the one evaluated by Slamecka when testing for parallelism in the vertical direction, is simply the number of items forgotten per unit time, $m l$, which is given by

$$
m I=\frac{\Delta Y_{i}}{T_{2}-T_{1}},
$$

where $\Delta Y_{i}$ represents the change in an absolute measure of performance (i.e., number of items recalled) in condition $i$ over the interval of time ranging from $T_{1}$ to $T_{2}$. If the number of items retained as a function of time could be described by a straight line, then Equation 1 would simply represent the slope of that line. For curvilinear forgetting functions, however, the value of $m I$ changes on a momentary basis and is given by the function's first derivative. In the case of exponential functions, $Y=a e^{-b T}$, for example, the first derivative $(d Y / d T)$ is described by the following equation:

$$
d Y / d T=-b a e^{-b T} .
$$

This equation describes the ever-changing slope of the forgetting function. When $T$ is small, the derivative has a relatively large negative value reflecting a steep downward slope. When $T$ is large, the value of the derivative is small, indicating a relatively flat function that is approaching its asymptote. If one forgetting function has a more negative first derivative than another for all $T$, then it always reflects a greater number of items forgotten per unit time and may therefore be said to exhibit a faster rate of forgetting. If the first derivatives are equal, then the two functions may be said to exhibit the same rate of forgetting.

It should be clear that if two curvilinear forgetting functions do happen to be equal with respect to $m l$ for all $T$, then they must arrive at different asymptotes. Except in the case of overlapping functions, equality with respect to $m l$ implies that one function is vertically offset from the other (i.e., they are vertically parallel). Therefore, if one function can be represented by $Y_{1}=a e^{-b t}$ (which declines to an asymptote of zero), the other, vertically parallel function must be represented by $Y_{2}=a e^{-b t}+c$, where $c$ represents an asymptote greater than zero. However, it is possible that, based on data from relatively short retention intervals, a function's predicted asymptote would fall below zero. Because a performance measure obviously cannot be negative, in that case vertical parallelism would not exist for all $T$, but only up to the moment at which the lower function declined to zero.

The second property of forgetting in which time appears in the denominator might be referred to as the proportional change in performance with respect to time, $m 2$, and is given by

$$
m 2=\frac{\Delta Y_{i}^{\prime}}{T_{2}-T_{1}},
$$

where $Y_{i}^{\prime}$ represents the proportion of the to-be-forgotten items in condition $i$ that are still retained. For example, consider the forgetting function described by the equation $Y=20 e^{-b T}+10$. According to this function, performance declines from an initial level of 30 items recalled (at $T=0$ ) to an eventual asymptotic level of 10 items recalled. One way to represent the course of forgetting in a situation such as this is to plot the proportion of the 20 to-be-forgotten items that are still retained at a given point in time. Initially, all 20 of these forgettable items will be recalled, and at that point $Y^{\prime}$ is equal to 1.0. At some later time, only 10 of these items will be retained (i.e., $Y^{\prime}=0.50$ ), and still later none of them will be retained (i.e., $Y^{\prime}=0$ ). At that point, performance will be at its asymptote of 10 items recalled.

For a function of the form $Y=a e^{-b T}+c$, where $Y$ represents the absolute number of items retained, the proportion of tobe-forgotten items still retained, $Y^{\prime}$, is $(Y-c) / a$. Therefore, the relationship between $Y^{\prime}$ and time is described by the equation, $Y^{\prime}=e^{-b T}$. As with $m 1$, the value of $m 2$ for this function changes continuously and is given by the function's first derivative $\left(d Y^{\prime} / d T\right)$,

$$
d Y^{\prime} / d T=-b e^{-b T} .
$$

Clearly, if two exponential functions have the same decay constants, they will exhibit the same rate of forgetting according to this measure (even if the two functions differ with respect $a$ or $c$ ).

In the previous example, absolute performance ranged from 30 items initially recalled to an asymptotic level of 10 items recalled. In general, however, the theoretical range of performance will not be known unless a very long retention interval is used. On the other hand, if we assume that absolute performance usually declines to an asymptote of zero, then the number of to-be-forgotten items and the number of items initially retained are one and the same. In that case, $m 2$ represents the slope of a forgetting function in which $Y^{\prime}$ is the number of items retained relative to the number originally retained (i.e., $Y^{\prime}=Y / a$ ). Occasionally, memory researchers have preferred to examine their forgetting functions in this way (Underwood \& Keppel, 1963). Incidentally if forgetting functions do always decline to an asymptote of zero, then they could not be vertically parallel for all $T$. 


\section{Time Over Performance}

Two additional properties of empirical forgetting functions involve the amount of time required to fall through a range of performance measured either on an absolute or on a relative basis. That is, unlike the two measures considered thus far, time appears in the numerator and the dependent measure of interest in the denominator. Loftus's test for horizontal parallelism evaluates forgetting functions on the basis of one such measure. More specifically, two functions are parallel in the horizontal direction if they require the same amount of time to fall through any arbitrarily selected absolute range of performance. For example, the forgetting functions shown in Figure 2 require exactly the same amount of time to decrease, say, from four items recalled to two items recalled. This measure of forgetting, $m 3$, is given by

$$
m 3=\frac{\Delta T_{i}}{Y_{2}-Y_{1}},
$$

where $\Delta T_{i}$ represents the amount of time required in condition $i$ to fall through a particular range of absolute performance bounded by the values $Y_{1}$ and $Y_{2}$. According to this new measure of forgetting, if one function requires more time to fall through a particular range of performance than another, then that function may be said to represent a slower rate of forgetting. If two functions require the same amount of time to fall through a particular range of performance, then they may be said to represent the same rate of forgetting. Furthermore, these conclusions will survive any monotonic transformation of the dependent measure because the same quantity, $Y_{2}-Y_{1}$, appears in the denominator of $m 3$ of both functions being compared. Hence, regardless of how $Y$ is transformed, the effect on $m 3$ will be the same in both cases.

If the equation describing performance as a function of time can be written $Y=a e^{-b T}+c$, then time as a function of performance is $T=-(1 / b) \log ((Y-c) / a)\}$, which is the same equation solved for $T$ instead of $Y$. The value of $m 3$ for this function depends on the level of performance $(Y)$ and once again is given by the function's first derivative,

$$
d T / d Y=-1 /\{b(Y-c)\} .
$$

This function reveals that if two exponential functions have the same decay constant $(b)$ and arrive at the same asymptote (c), then they will be horizontally parallel (regardless of the value of $a$ ). However, if two functions have the same decay constant $(b)$ but arrive at different asymptotes $(c 1$ and $c 2)$, then they will not be parallel in the horizontal direction (i.e., they will not be equal with respect to $m 3$ ). In general, a function associated with a more negative value of $d T / d Y$ requires more time to fall through a particular range of performance and may therefore be said to exhibit a slower rate of forgetting.

The fourth and final property of empirical forgetting functions to be considered is the amount of time required to fall through a proportional range of performance, $m 4$, which is given by

$$
m 4=\frac{\Delta T_{i}}{Y_{2}^{\prime}-Y_{1}^{\prime}}
$$

If two functions require the same amount of time to fall through the range of performance bounded by $Y_{1}^{\prime}$ and $Y_{2}^{\prime}$, then they may be said to exhibit the same rate of forgetting. If one function requires more time to fall through a particular proportion of its range, then that function may be said to exhibit a slower rate of forgetting.

As indicated earlier, an exponential forgetting function expressed in proportional terms is given by $Y^{\prime}=e^{-b T}$. Solving this equation for $T$ instead of $Y$ yields the equation $T=-(1 /$ b) $\log \left(Y^{\prime}\right)$. The value of $m 4$ for this function depends on $Y^{\prime}$ and is described by the function's first derivative,

$$
d T / d Y^{\prime}=-1 /\left(b Y^{\prime}\right) .
$$

From this equation it is clear that if two exponential functions have the same decay constant $(b)$, they will be equal with respect to $m 4$ (even if they differ with respect to $a$ and $c$ ). In that sense, $m 4$ and $m 2$ are alike.

Of special interest is the amount of time required to fall through the proportional range of 1.0 to 0.5 , because that value represents the half-life of forgetting. In the example considered earlier, in which performance decreased from 30 items recalled initially to an asymptotic level of 10 items recalled, the half-life would be the amount of time required to forget 10 of the 20 to-be-forgotten items. For forgetting functions that decline to an asymptote of zero, the half-life is simply the time required to fall to one half of initial performance. In either case, the half-life of exponential functions is provided by integrating Equation 8 over the range of 1.0 to 0.5 and is equal to $-\log (0.5) / b$ (or simply $0.693 / b$ ). Indeed, for exponential functions, the amount of time required to fall from any $Y^{\prime}{ }_{i}$ to $0.5 Y^{\prime}{ }_{i}$ is always equal to $0.693 / b$.

\section{The Rate of Forgetting}

At various times, different investigators have chosen one of these four properties on which to base their conclusions about the rate of forgetting. Those concerned with the absolute change in performance per unit time $(m I)$ test for parallelism in the vertical direction. Those concerned with the amount of time required to fall through a given range of performance $(m 3)$ test for parallelism in the horizontal direction. Those concerned with the proportional decay per unit time $(m 2)$, or the half-life of forgetting $(m 4)$, typically fit mathematical functions to evaluate changes in the decay constant (Wickelgren, 1972). Because any of these properties might be used to support conclusions about the rate of forgetting, an example of how they can seem to disagree may be worth considering.

Figure 3 depicts two hypothetical forgetting functions. The upper curve, which we may assume is associated with the higher degree of learning, is described by the equation $Y=$ $6 e^{-0.5 T}+4$ and the lower curve by $Y=4 e^{-0.5 T}+1$. As described by Equation 2, the first derivative of the upper function is $-3 e^{-0.5 T}$, whereas the first derivative of the lower function is $-2 e^{-0.5 T}$. In other words, for all $T$, the first function exhibits a steeper downward slope than the second. Therefore, if we base our conclusions about the rate of forgetting on $m l$, then a higher degree of learning results in a faster rate of forgetting. On the other hand, according to Equation 6 , the value of $m 3$ for the upper function is $-2 /(Y-4)$, whereas the 


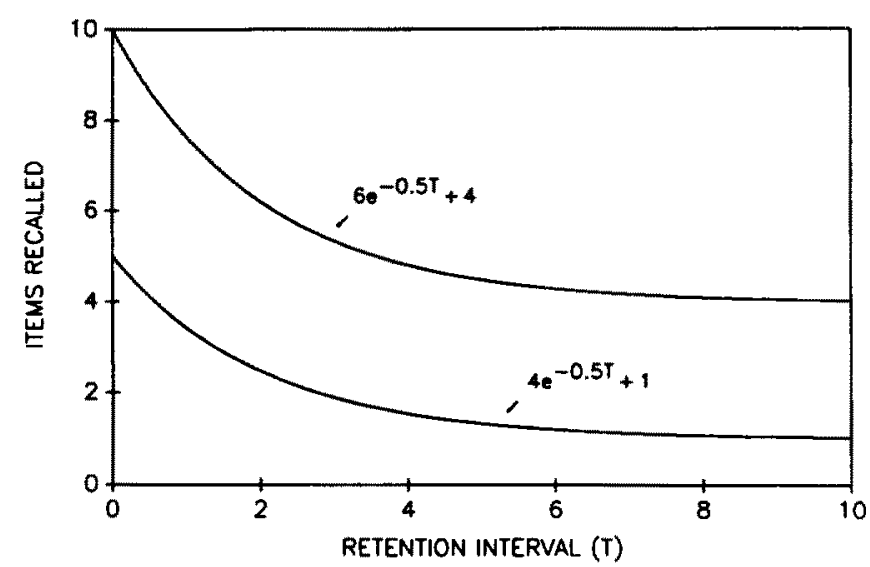

Figure 3. Idealized exponential forgetting functions.

corresponding value for the lower function is $-2 /(Y-1)$. Thus, if we instead refer to this property as rate of forgetting, we will reach the opposite conclusion that a higher degree of learning results in a slower rate of forgetting. However, the decay constants associated with each function are identical (viz., 0.5), which means that they are equal with respect to both $m 2$ and $m 4$. As described by Equation 4 , the value of $m 2$ for both functions is $-0.5 e^{-0.5 T}$, and, as described by Equation 8 , the value of $m 4$ for both functions is $-2 / Y^{\prime}$. Therefore, if we choose to emphasize either of these properties, we will reach the conclusion that the degree of learning is independent of the rate of forgetting.

Obviously, I have chosen an example that, when coupled with vague phrasing about the rate of forgetting, appears to yield a confused and conflicting picture indeed. In fact, I have simply considered four different properties of empirical forgetting functions: (a) absolute change in performance with respect to time, (b) proportional change in performance with respect to time, (c) elapsed time with respect to absolute change in performance, and (d) elapsed time with respect to proportional change in performance. The question is whether a state of disagreement exists when conclusions based on $m l$ differ from those based on $m 2, m 3$, or $m 4$. The answer seems to be no. If those properties change in different ways when the degree of learning is manipulated, they cannot be said to be in conflict. Similarly, we would not be especially convinced of our conclusions if all four methods agreed that a higher degree of learning results in a slower rate of forgetting. They address different empirical properties and so neither reinforce nor contradict each other.

From this point of view, Loftus and Slamecka have no disagreement about whether the degree of learning influences the rate of forgetting. On the other hand, they clearly disagree about the less important matter of which property ought to be called rate of forgetting and the much more important matter of the extent to which we should concern ourselves with the issue of scale. As argued later, the latter issue depends on whether one's objective is to test a psychological model of memory or to establish an empirical principle of forgetting. Before addressing that issue, however, a new perspective on the analysis of forgetting functions recently offered by Bogartz
(1990) is considered. His approach, which is based on Anderson's (1982) functional measurement theory, attempts to describe psychological forgetting functions in contrast to the empirical forgetting functions considered to this point. These psychological functions can still be evaluated with respect to any of the four properties considered thus far to decide whether a higher degree of learning results in a slower rate of forgetting.

\section{Psychological Time}

As indicated earlier, the essence of the scaling problem is that our performance measure is presumably only monotonically related to an underlying psychological variable. Bogartz (1990) argued that, in addition, our measures of both degree of learning and time are related to their psychological counterparts in the same way. To illustrate this idea, consider the simple model $r=o e^{-t}$. This model consists of psychological variables only and states that information retained $(r)$ is equal to the degree of learning $(o)$ multiplied by an exponential function of what we might call "psychological time" $(t)$. All three of these psychological constructs $(r, o$, and $t)$ are assumed to be monotonically related to physical measurements such as number of items recalled $(R)$, number of learning trials $(O)$, and elapsed time $(T)$.

Bogartz (1990) evaluated the psychological model presented above using data from Hellyer (1962). In this experiment, subjects were exposed to multiple learning trials (ranging from 1 to 8) followed by a retention interval ranging from 3 to 27 s. The strategy for testing this model involves searching for monotonic transformations of $O$ and $T$, which, when substituted into the psychological model as $o$ and $t$, yield values of $r$ that are monotonically related to the obtained recall scores $(R)$. For example, Bogartz found that if the actual $O$ values $(1,2,4$, or 8 learning trials) were monotonically rewritten as 2.7, 3.3, 4.4, and 5.8 learning trials and the actual retention intervals $(3,9,18$, and $27 \mathrm{~s})$ were monotonically rewritten as $1,1.8,2.0$, and $2.1 \mathrm{~s}$, then when the transformed values were plugged back into the model they yielded values of $r$ that were indeed monotonically related to the observed data, $R$. Thus, Bogartz concluded that the data supported the proposed psychological model.

The model under consideration, $r=o e^{-t}$, might be regarded as a psychological forgetting function as opposed to the empirical forgetting functions considered thus far. To evaluate the relationship between the degree of learning and the rate of forgetting, the psychological function can be examined with respect to any of the four properties considered earlier. The first derivative of this function $(\mathrm{ml})$ represents the absolute rate of change in information retained $(r)$ with respect to psychological time $(t)$. As noted by Bogartz, his psychological model predicts that a higher degree of learning will result in a faster rate of forgetting according to this measure because the model's first derivative is equal to $-o e^{-t}$. Thus, the higher the degree of learning, the more rapid the rate of change in $r$. On the other hand, the half-life of this function and its proportional rate of change with respect to time are independent of $o$. The half-life is $.69 / 1$, or 0.69 units of psychological time (regardless of the value of $o$ ), and the proportional 
rate of change is given by $-e^{-1}$, which again is independent of $o$. According to these measures, therefore, the rate of forgetting is not influenced by the degree of learning.

Bogartz (1990) chose to base his conclusions about the rate of forgetting on the latter measures and therefore suggested that his model "supports Slamecka's conclusion that rate of forgetting does not depend on original learning" (p. 144). However, it might be more accurate to state that they neither agree nor disagree about that because they are not talking about the same property. Even if we ignore the differences between empirical and psychological forgetting functions, Bogartz based his conclusion on a measure of proportional change, whereas Slamecka based his on a measure of absolute change. As such, their conclusions do not agree and certainly do not reinforce or support each other.

Bogartz arrived at his conclusions about the effects of learning on forgetting after evaluating a single psychological model, $\alpha e^{-t}$, and remarked that "the perfect fit of the model to Hellyer's data increases credence in the scale transformations as revealing how the measured variables relate to the underlying variables" (p. 141). However, it should be added that Hellyer's data also support several additional psychological models that convey different conclusions about the effects of learning on the rate of forgetting. For example, the models $e^{-t / 0}, o e^{-t / 0}$, and $e^{-t}+o$ are all capable of yielding data that satisfy the criteria used to support the model, $o e^{-t}$. In each case, monotonic transformations of $O$ and $T$ can be found, which, when substituted into one of these models, yield numbers monotonically related to Hellyer's empirical data, $R$. Hellyer's data were presented by Bogartz (1990), and the appropriate transformations of $T$ and $O$ for each of the new models proposed here are shown in Table 1. These transformations were determined by a computer program that generated two sets of four random numbers (representing tentative values for $t$ and $o$ ), which were then used to produce a table of predicted $r$ values. For each model, this procedure was repeated until the ordinal pattern of the predicted values was the same as the ordinal pattern of observed $R$ values.

The model tested by Bogartz implies that a higher degree of learning results in a faster rate of forgetting according to $m l$, but has no effect on the rate of forgetting according to $m 2, m 3$, or $m 4$. Each of the new models presented in Table 1 suggests a different interpretation. For example, the model $o e^{-t / o}$ implies that a higher degree of learning increases the rate of forgetting according to $m l$, has a variable effect on $m 2$, and decreases the rate of forgetting according to $m 3$ and $m 4$. Thus, although Hellyer's data are useful for illustrating the application of functional measurement theory to the question of forgetting, it may be premature to suggest that those data can support any conclusions about the effects of learning on forgetting. On the other hand, a more elaborate experimental design involving a greater number of retention intervals and degrees of learning might be able to differentiate more effectively between competing alternatives.

Unlike the strategies considered earlier, Bogartz's approach does not concern itself with analyzing specific properties of empirical forgetting functions. Instead, both the independent and dependent variables are transformed until it is determined that they can (or cannot) conform to the proposed model. The approach highlights the essential issue in the debate over
Table 1

Monotonic Transformations of Time and Original Learning for Three Psychological Models of Forgetting

\begin{tabular}{|c|c|c|c|c|c|}
\hline \multicolumn{2}{|c|}{$e^{-t / o}$} & \multicolumn{2}{|c|}{$\alpha e^{-t / o}$} & \multicolumn{2}{|c|}{$e^{-t}+o$} \\
\hline$t$ & $o$ & $t$ & $o$ & $t$ & $o$ \\
\hline 0.08 & 0.22 & 0.22 & 1.94 & 0.28 & 0.29 \\
\hline 0.36 & 0.31 & 0.68 & 2.03 & 1.09 & 0.35 \\
\hline 0.61 & 0.56 & 0.82 & 2.20 & 1.76 & 0.53 \\
\hline 0.74 & 0.99 & 0.93 & 2.32 & 1.99 & 0.71 \\
\hline
\end{tabular}

Note. The values of $t$ represent monotonic transformations of $T(3$, $9,18$, and $27 \mathrm{~s})$, and the values of $o$ represent monotonic transformations of $O(1,2,4$, and 8 trials).

how to analyze the course of forgetting: Loftus and Bogartz are concerned (to different degrees) with psychological constructs or variables; Slamecka is not.

\section{Psychological Models Versus Empirical Principles}

Loftus and Bogartz are both of the opinion that Slamecka's empirical view of forgetting entails an implicit psychological model that he chooses not to articulate, and for that reason the scaling problem simply cannot be avoided. One reason for their position is that Slamecka accepted the existence of floor and ceiling effects in forgetting functions and therefore tacitly acknowledged a theoretical construct that is imperfectly gauged by the dependent measure in use. Slamecka, on the other hand, chose to think of forgetting functions in empirical terms, without necessarily adopting any theoretical stance about forgetting, unarticulated or otherwise.

The semantic debate over the proper use and interpretation of terms such as forgetting has a long history in the field of philosophy (e.g., Ryle, 1959; Wittgenstein, 1953; Wood \& Pitcher, 1970), and I do not try to resolve it here. Nevertheless, it might be useful to contrast two reasonable interpretations of the term and to consider how those interpretations help guide the analysis of forgetting functions. That the term forgetting can be used to refer to changes in a psychological variable with respect to time seems to be a relatively uncontroversial position. Thus, for example, one might say that forgetting has occurred when the strength of a memory trace (or the amount of information retained) has declined over time. From another point of view, however, the term forgetting may refer to changes in the ability to evidence the effects of past experience. Thus, for example, we might say that I have forgotten the "Star Spangled Banner" if my ability to recite its lines is no longer as accurate as it once was. To construe the term in this way, however, is not to deny that memory performance is subserved by dynamic processes occurring on another level.

Although they might differ about its true meaning, advocates of either of these interpretations will often use the term forgetting under the same set of conditions. For example, when a measure such as the number of words recalled is changing with respect to time, most observers would probably agree that some forgetting has occurred. With regard to the interpretation of floor and ceiling effects, however, the applicability of the term forgetting is less certain. 


\section{Floor and Ceiling Effects}

Consider a situation in which recognition of faces is perfectly accurate even after a long retention interval. From a psychological standpoint, one could assume that some forgetting has nevertheless occurred. That is, perhaps the memory strengths of the individual faces has weakened but not enough to be reflected by the chosen dependent measure. From a performance perspective, by contrast, the term forgetting would not apply because the faces can be identified as readily as ever (although it may indeed be true that their memory traces have weakened in strength). When the measure does eventually begin to exhibit change, then at that point forgetting is said to have occurred.

Advocates of either interpretation of forgetting would probably agree that, unless a performance measure changes with respect to time, the data are of little use in helping to establish theories or principles of forgetting. However, from a psychological standpoint, a model based on such a measure is not conceptually confined to the period of time during which the performance measures undergo change. For example, the forgetting function from the low degree of learning condition might be on the floor while the corresponding function from the high degree of learning condition is still decreasing. That result would not necessarily damage a psychological model that implies equal forgetting rates, because the underlying memory traces in both conditions might still be weakening at the same rate. Unlike a psychological model, however, an empirical principle of forgetting always presupposes behavioral transition. From this point of view, to compare one function that declines over time with another that is static with respect to time is to compare forgetting with the absence of forgetting. Indeed, by definition, an empirical principle of forgetting applies only when the measures under consideration are simultaneously in a state of transition. When one or both measures are on the ceiling or floor, the principle no longer applies.

Although an empirical analysis must avoid floor and ceiling effects, it might be argued that Slamecka and McElree's (1983) decision to avoid analyzing data that appeared to be approaching the floor may be unwarranted. If, after taking measurement error into consideration, two functions are still decreasing with respect to time, then forgetting is still occurring and the data should not be excluded (Loftus, 1985b). In some cases (e.g., Krueger, 1929), the inclusion of data reflecting low levels of recall would suggest that forgetting functions are not vertically parallel after all. However, because the necessary statistical analyses were not performed in the older studies reviewed by Slamecka and McElree (1983), identifying the onset of a floor effect is necessarily somewhat arbitrary.

\section{Research Objectives}

Depending on how the term forgetting is initially interpreted, research on the course of forgetting will probably be concerned with identifying either a psychological model or an empirical principle. The appropriate analysis of forgetting functions depends, in part, on which of these objectives one has in mind. Obviously (thanks to Loftus), if one sets out to characterize the temporal properties of a psychological model of memory, then the question of how the chosen dependeni measure maps onto the process of interest must be confronted. In that case, testing functions for parallelism in the horizontal direction or evaluating the viability of psychological models such as $o e^{-t}$ may be the appropriate strategies. In either case, the use of a single dependent measure (e.g., number of items recalled) may be sufficient. However, if one's objective is to identify the limits of empirical reliability, then a different strategy is in order. Essentially, that strategy involves testing whether conclusions based on one measure of memory in one situation are consistent with those based on other measures of memory in other situations. To the extent that empirical reliability is observed, broad-based laws of memory performance may be established.

The possibility that different empirical measures of memory will behave in inconsistent ways obviously cannot be ruled out. Indeed, some degree of inconsistency among the various measures seems almost certain. Nevertheless, even if a monolithic principle of memory performance is unlikely ever to be identified, it might be possible to discover principles that hold within certain identifiable domains. Thus, for example, the degree of learning may be unrelated to the rate of forgetting when meaningful material is used, and may reliably affect the rate of forgetting when nonsense syllables are used. Under a more pessimistic scenario, however, no empirical reliability whatsoever will be found. In that case, perhaps the only level at which order will be established is at the level of the psychological model (although, as illustrated earlier, even this is by no means guaranteed).

On the other hand, one notable feature of most empirical forgetting functions is their apparent similarity. Klatzky $(1980$, p. 276$)$, for example, commented on the similarity in the form of forgetting functions as diverse as those based on savings measured over a period of weeks and those based on free recall measured over a period of seconds. More to the point, Slamecka and McElree (1983) found that a higher degree of learning did not alter the rate of forgetting $(\mathrm{ml})$ based on free recall of categorized words, cued recall of highimagery words, gist recall for sentences, and semantic recognition for those same sentences. If the same result were consistently found for other to-be-remembered materials and other measures of memory, we might be reasonably confident that a general empirical principle of forgetting had been identified. In addition, if that principle were also supported by experiments using more ecologically valid procedures, we might even be bold enough to claim that the principle holds for extralaboratory memory performance as well. As indicated earlier, however, such a principle would only apply to performance measures in a state of transition and not to underlying psychological variables.

\section{The Mathematics of Forgetting}

The empirical analysis of forgetting functions may not be especially well served by the three analytical strategies considered to this point. These strategies entail testing for vertical parallelism, testing for horizontal parallelism, and monotonically transforming the independent and dependent measures to test a psychological model. A fourth strategy that has not 
been extensively pursued in recent years involves attempting to identify the mathematical form of empirical forgetting curves. Of these strategies, the latter may be able to provide the most complete description of the empirical course of forgetting. Indeed, questions about the rate of forgetting are inherently mathematical, and closer attention to the mathematics of empirical forgetting functions may be able to provide the clearest possible answers (White, in press).

Wickelgren $(1972,1974)$ was the last to investigate systematically the mathematical form of forgetting functions in humans. In a series of experiments, Wickelgren (1972) tested recognition memory for word pairs over a variety of retention intervals and then converted the raw data into $d^{\prime}$ scores (Green \& Swets, 1966). An equation of the form $d^{\prime}=$ $a e^{-b \sqrt{T}}$ described his data more accurately than several reasonable alternatives, including linear, simple exponential, power, and logarithmic decay functions. In these experiments, $d^{\prime}$ was used as a dependent measure because, given the assumptions of signal detection theory, that value provides a linear scale upon which to measure a psychological variable such as trace strength. Nevertheless, as illustrated later, the same mathematical strategy can be used to quantify the effects of learning on the rate of forgetting based on a variety of empirical measures. Although these measures may not be assumed to provide a linear scale of an underlying memory trace, they may be found to vary in consistent ways according to the various definitions of forgetting rate considered earlier.

When the function $a e^{-b \sqrt{T}}$ was fitted to the forgetting data of individual subjects, Wickelgren found that the decay parameter, $b$, reliably decreased and that initial performance, $a$, reliably increased with higher degrees of learning. To the extent that this exponential power function accurately describes the course of forgetting, this information is sufficient to reveal the effects of original learning on $m 1, m 2, m 3$, and $m 4$. By contrast, if we found that two forgetting functions were nonparallel in the horizontal direction, we would be in a position to rule out equality with respect to $m 3$ only and could draw no conclusions based on $m 1, m 2$, or $m 4$. Similarly, if we found that two forgetting functions were nonparallel in the vertical direction, we would be in a position to rule out equality with respect to $\mathrm{ml}$ only and would be unable to draw any conclusions about $m 2, m 3$, or $m 4$. Moreover, although a test for vertical parallelism may be an effective way to reject the hypothesis of identical slopes, it may not be the best way to support the hypothesis that two forgetting functions have equivalent slopes (an approach that requires acceptance of the null hypothesis). The principal advantage of a mathematical analysis is that the parameters of forgetting can be directly quantified. As such, systematic changes in the magnitude of those parameters, such as the decay constant, are more clearly revealed.

As an example, consider some data recently reported by Slamecka and Katsaiti (1988). In a control condition of their Experiment 1, subjects were exposed to one of three degrees of learning followed, in each case, by three retention tests. Their results (estimated from their Figure 1) are reproduced in Figure 4. Because only three retention intervals were used, the actual mathematical form of these functions obviously cannot be determined. Nevertheless, it is instructive to fit
Wickelgren's exponential power function to these data and to examine the effects of learning on its two parameters. The exponential power function was fitted to the data using a nonlinear regression algorithm. As shown in Figure 4, the trends in the fitted functions are the same as those observed by Wickelgren in his analysis of $d^{\prime}$ recognition scores. That is, the decay parameter, $b$, systematically decreases, whereas the value of $a$ increases with higher degrees of learning. The same trends were found when the equation was modified to allow for the possibility of different asymptotes (a requirement of vertically parallel functions). Thus, to the extent that this function describes the course of forgetting, these trends encourage a cautious attitude toward the acceptance of a null hypothesis stating that the slopes of these functions are unaffected by the degree of learning.

Attention to the mathematics of forgetting may provide the most straightforward way to isolate the effects of learning on $m 1, m 2, m 3$, and $m 4$, but the approach also imposes certain procedural requirements. First, and most important, a relatively large number of data points must be collected to reveal the mathematical form of a forgetting function (and, perhaps, to reveal the point at which the data leave the ceiling). Given the curvilinear nature of most forgetting functions, the use of three or even four different retention intervals is probably insufficient. By contrast, tests for parallelism in either direc. tion can be performed when as few as two retention intervals are used.

Second, a mathematical strategy is most efficiently applied at the individual level, which means that each subject must be run for multiple sessions to achieve smooth forgetting curves. In that way, the consistency with which, say, the decay parameter changes with increases in the degree of learning can be readily determined (cf. Wickelgren, 1972). In spite of difficulties such as these, however, the mathematical analysis of empirical forgetting functions represents an important

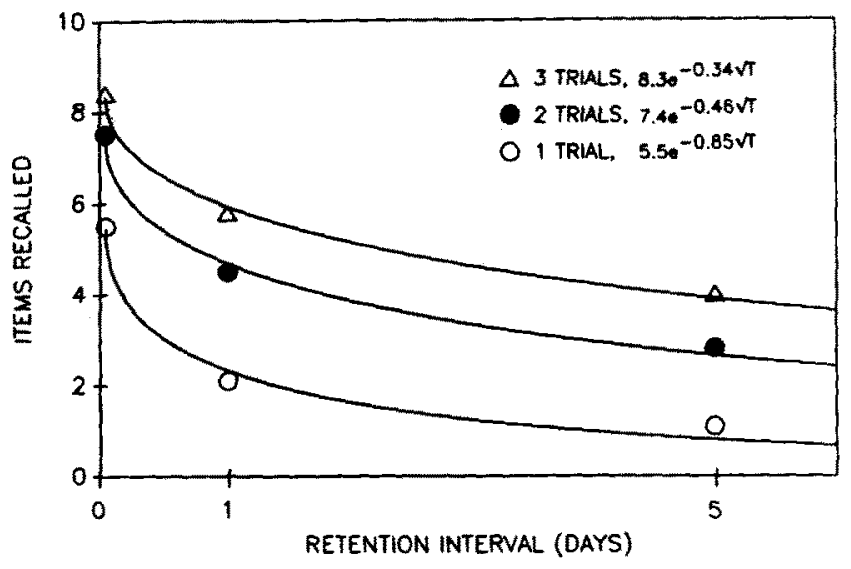

Figure 4. Number of words recalled as a function of the retention interval for three degrees of learning. (The solid curves represent the least squares fit of the function $a e^{-b \sqrt{\tau}}$. The data were taken from "Normal Forgetting of Verbal Lists as a Function of Prior Testing" by N. J. Slamecka and L. T. Katsaiti, 1988, Journal of Experimental Psychology: Learning, Memory, and Cognition, 14, p. 720. Copyright 1988 by the American Psychological Association. Adapted by permission.) 
alternative to the methods based on parallelism or data transformations.

\section{Conclusion}

To adopt an empirical position on the issues reviewed in this article is not to deny that the observed behavioral process of forgetting is a reflection of some underlying biological or psychological process that may also be referred to as forgetting. However, the essential question concerns which process we propose to take hold of. If we attempt to identify the temporal properties of a model of memory, then the way in which the chosen dependent measure maps onto that process must be considered. If we instead attempt to identify empirical principles of forgetting, then the ways in which various dependent measures change as a function of the degree of learning must be determined.

Each of these approaches has some important limitations from the perspective of the other. For example, even if a psychological model can be identified, that achievement will not facilitate any predictions about the rate of forgetting based on a performance measure such as $m l$. Thus, if we find that our forgetting curves can be monotonically transformed to accord with a model such as $o e^{-t}$, we cannot then use this information to conclude that, in terms of actual performance, the absolute rate of change with respect to time will, in general, be influenced by the degree of learning. We simply do not know because the dependent measure we choose to use may or may not be linearly related to the relevant psychological variable.

Similarly, no matter how reliable the various empirical measures of memory happen to be, we cannot use an established principle of performance to draw any conclusions about the temporal characteristics of an underlying psychological model or construct. The reason, again, is that the function relating a dependent measure to a psychological variable is usually unknown. Indeed, it is this very fact that prompted Loftus to challenge Slamecka and McElree's (1983) original conclusions about the rate of forgetting. Based on considerations such as these, it seems clear that the proper approach to analyzing the course of forgetting is largely determined by the objectives one has in mind.

\section{References}

Anderson, N. H. (1982). Methods of information integration theory. San Diego, CA: Academic Press.

Bahrick, H. P. (1984). Semantic memory content in permastore: Fifty years of memory for Spanish learned in school. Journal of Experimental Psychology: General, 113, 1-47.

Bogartz, R. S. (1990). Evaluating forgetting curves psychologically. Journal of Experimental Psychology: Learning, Memory, and Cognition, $16,138-148$

Green, D. M., \& Swets, J. A. (1966). Signal detection theory and psychophysics. New York: Wiley.
Hellyer, S. (1962). Frequency of stimulus presentation and shortterm decrement in recall. Journal of Experimental Psychology, 64, 650.

Klatzky, R. M. (1980). Human memory: Structures and processes (2nd ed.). San Francisco: W. H. Freeman.

Krantz, D. H. \& Tversky, A. (1971). Conjoint-measurement analysis of composition rules in psychology. Psychological Review, 78, 151169.

Krueger, W. C. F. (1929). The effects of overlearning on retention. Journal of Experimental Psychology, 12, 71-78.

Loftus, G. R. (1978). On interpretation of interactions. Memory and Cognition, 6, 312-319.

Loftus, G. R. (1985a). Evaluating forgetting curves. Journal of Experimental Psychology: Learning, Memory, and Cognition, 11, 397-406.

Loftus, G. R. (1985b). Consistency and confoundings: Reply to Slamecka. Journal of Experimental Psychology: Learning, Memory, and Cognition, 11, 817-820.

Modigliani, V. (1976). Effects on a later recall by delaying initial recall. Journal of Experimental Psychology: Human Learning and Memory, 2, 609-622.

Rundquist, W. N. (1983). Some effects of remembering on forgetting. Memory and Cognition, $11,641-650$.

Rundquist, W. N. (1986). Changes in the rate of forgetting produced by recall tests. Canadian Journal of Psychology, 40, 282-289.

Ryle, G. (1949). The concept of mind. New York: Barnes \& Noble.

Slamecka, N. J. (1985). On comparing rates of forgetting: Comment on Loftus (1985). Journal of Experimental Psychology: Learning, Memory, and Cognition, 11, 812-816.

Slamecka, N. J., \& Katsaiti, L. T. (1988). Normal forgetting of verbal lists as a function of prior testing. Journal of Experimental Psychology: Learning, Memory, and Cognition, 14, 716-727.

Slamecka, N. J., \& McElree, B. (1983). Normal forgetting of verbal lists as a function of their degree of learning. Joumal of Experimental Psychology: Learning, Memory, and Cognition, 9, 384-397.

Squire, L. R. (1989). On the course of forgetting in very long-term memory. Journal of Experimental Psychology: Learning, Memory, and Cognition. 15, 241-245.

Underwood, B. J. (1964). Degree of learning and the measurement of forgetting. Journal of Verbal Learning and Verbal Behavior, 3 , 112-129.

Underwood, B. J., \& Keppel, G. (1963). Retention as a function of degree of learning and letter-sequence interference. Psychological Monographs, 77 (1, Whole No. 567).

White, K. G. (in press). Psychophysics of direct remembering. In J. A. Commons, M. C. Davison, \& J. A. Nevin (Eds.), Quantitative analyses of Behavior: Vol. II. Signal Detection. Hillsdale, NJ: Erlbaum.

Wickelgren, W. A. (1972). Trace resistance and the decay of longterm memory. Journal of Mathematical Psychology, 9, 418-455.

Wickelgren, W. A. (1974). Single-trace fragility theory of memory dynamics. Memory and Cognition, 2, 775-780.

Wittgenstein, L. (1953). Philosophical investigations (3rd ed.) (G.E.M. Anscombe, Trans.). New York: Macmillan.

Wood, O. P., \& Pitcher, G. (Eds.). (1970). Ryle: A collection of critical essays. Garden City, NY: Anchor Books.

Received October 6, 1989

Revision received January 16, 1990 Accepted January 18, 1990 